UUITP-13/96

OUTP-96-29P

hep-ph/9607237

\title{
Indirect Neutralino Detection Rates in Neutrino Telescopes
}

\author{
Lars Bergström Joakim Edsjȫ \\ Department of Theoretical Physics, Uppsala University, \\ Box 803, SE-751 08 Uppsala, Sweden \\ Paolo Gondolof \\ Department of Physics, University of Oxford, \\ 1 Keble Road, Oxford, OX1 3NP, United Kingdom
}

\begin{abstract}
Neutralinos annihilating in the center of the Sun or the Earth may give rise to a detectable signal of neutrinos. We derive the indirect detection rates for neutrino telescopes in the minimal supersymmetric extension of the standard model. We show that even after imposing all phenomenological and experimental constraints that make the theories viable, regions of parameter space exist which can already be probed by existing neutrino telescopes. We compare with the discovery potential of supersymmetry at LEP2 as well as direct detections and point out the complementarity of the methods.
\end{abstract}

\section{Introduction}

Supersymmetric neutralinos with masses in the GeV-TeV range are among the leading non-baryonic candidates for the dark matter in our galactic halo. One of the most

*Permanent address: Department of Physics, Stockholm University, Box 6730, SE-113 85 Stockholm, Sweden; E-mail address: lbe@physto.se

${ }^{\dagger}$ E-mail address: edsjo@teorfys.uu.se

${ }_{\ddagger}^{\ddagger}$ E-mail address: p.gondolo1@physics.oxford.ac.uk 
promising methods for the discovery of neutralinos in the halo is via observation of energetic neutrinos from their annihilation in the Sun [1] and/or the Earth [2]. Through elastic scattering with the atomic nuclei in the Sun or the Earth, a neutralino from the halo can lose enough energy to remain gravitationally trapped [3]. Trapped neutralinos sink to the core of the Sun or the Earth where they annihilate into ordinary particles: leptons, quarks, gluons and - depending on the masses - Higgs and gauge bosons. Because of absorption in the solar or terrestrial medium, only neutrinos are capable of escaping to the surface. Neutralinos do not annihilate into neutrinos directly [4], but energetic neutrinos may be produced via hadronization and/or decay of the direct annihilation products. These energetic neutrinos may be discovered by terrestrial neutrino detectors.

In this letter, we consider Cerenkov neutrino telescopes. They consist of large underground arrays of photo-multipliers to detect the Čerenkov light emitted by muons generated in charged-current interactions of neutrinos with the medium surrounding the detector. Underground Cerenkov detectors, originally built to search for proton decay, have already started to explore (and constrain) neutralinos as dark matter candidates [5, 6]. A new generation of much larger neutrino telescopes, utilizing large volumes of natural water or ice (for a review, see [7]) is currently under construction, which will increase the sensitivity for high-energy neutrino point sources by an order of magnitude or more.

The prediction of muon rates is quite involved: we compute neutralino capture rates in the Sun and the Earth, fragmentation functions in basic annihilation processes, propagation through the solar or terrestrial medium, charged current cross sections and muon propagation in the rock, ice or water surrounding the detector. In addition, there may be scattering of the Čerenkov photons generated by the muons, due to impurities in the medium.

\section{Definition of the Supersymmetric Model}

The minimal $N=1$ supersymmetric extension of the standard model is defined by the following superpotential and soft supersymmetry-breaking potential (for notation and details, see Ref. [8])

$$
\begin{aligned}
W=\epsilon_{i j}\left(-\hat{\mathbf{e}}_{R}^{*} \mathbf{Y}_{E} \hat{\mathbf{l}}_{L}^{i} \hat{H}_{1}^{j}-\hat{\mathbf{d}}_{R}^{*} \mathbf{Y}_{D} \hat{\mathbf{q}}_{L}^{i} \hat{H}_{1}^{j}+\hat{\mathbf{u}}_{R}^{*} \mathbf{Y}_{U} \hat{\mathbf{q}}_{L}^{i} \hat{H}_{2}^{j}-\mu \hat{H}_{1}^{i} \hat{H}_{2}^{j}\right) \\
V_{\text {soft }}=\epsilon_{i j}\left(-\tilde{\mathbf{e}}_{R}^{*} \mathbf{A}_{E} \mathbf{Y}_{E} \tilde{\mathbf{l}}_{L}^{i} H_{1}^{j}-\tilde{\mathbf{d}}_{R}^{*} \mathbf{A}_{D} \mathbf{Y}_{D} \tilde{\mathbf{q}}_{L}^{i} H_{1}^{j}+\tilde{\mathbf{u}}_{R}^{*} \mathbf{A}_{U} \mathbf{Y}_{U} \tilde{\mathbf{q}}_{L}^{i} H_{2}^{j}\right. \\
\left.\quad-B \mu H_{1}^{i} H_{2}^{j}+\text { h.c. }\right) \\
\quad+H_{1}^{i *} m_{1}^{2} H_{1}^{i}+H_{2}^{i *} m_{2}^{2} H_{2}^{i} \\
+\tilde{\mathbf{q}}_{L}^{i *} \mathbf{M}_{Q}^{2} \tilde{\mathbf{q}}_{L}^{i}+\tilde{\mathbf{l}}_{L}^{i *} \mathbf{M}_{L}^{2} \tilde{\mathbf{l}}_{L}^{i}+\tilde{\mathbf{u}}_{R}^{*} \mathbf{M}_{U}^{2} \tilde{\mathbf{u}}_{R}+\tilde{\mathbf{d}}_{R}^{*} \mathbf{M}_{D}^{2} \tilde{\mathbf{d}}_{R}+\tilde{\mathbf{e}}_{R}^{*} \mathbf{M}_{E}^{2} \tilde{\mathbf{e}}_{R}
\end{aligned}
$$




$$
+\frac{1}{2} M_{1} \tilde{B} \tilde{B}+\frac{1}{2} M_{2}\left(\tilde{W}^{3} \tilde{W}^{3}+2 \tilde{W}^{+} \tilde{W}^{-}\right)+\frac{1}{2} M_{3} \tilde{g} \tilde{g} .
$$

Here $i$ and $j$ are SU(2) indices $\left(\epsilon_{12}=+1\right)$, Y's, A's and M's are $3 \times 3$ matrices in generation space, and the other boldface letters are vectors in generation space.

Electroweak symmetry breaking is caused by both $H_{1}^{1}$ and $H_{2}^{2}$ acquiring vacuum expectation values,

$$
\left\langle H_{1}^{1}\right\rangle=v_{1}, \quad\left\langle H_{2}^{2}\right\rangle=v_{2},
$$

with $g^{2}\left(v_{1}^{2}+v_{2}^{2}\right)=2 m_{W}^{2}$, with the further assumption that vacuum expectation values of all other scalar fields (in particular, squark and sleptons) vanish. This avoids color and/or charge breaking vacua.

To reduce the number of free parameters, we make simplifying unification assumptions for the gaugino mass parameters

$$
\begin{aligned}
& M_{1}=\frac{5}{3} \tan ^{2} \theta_{w} M_{2}, \\
& M_{2}=\frac{\alpha_{e m}}{\sin ^{2} \theta_{w} \alpha_{s}} M_{3},
\end{aligned}
$$

and a simple ansatz for the soft supersymmetry-breaking parameters in the sfermion sector:

$$
\begin{aligned}
\mathbf{M}_{Q} & =\mathbf{M}_{U}=\mathbf{M}_{D}=\mathbf{M}_{E}=\mathbf{M}_{L}=m_{0} \mathbf{1} \\
\mathbf{A}_{U} & =\operatorname{diag}\left(0,0, A_{t}\right) \\
\mathbf{A}_{D} & =\operatorname{diag}\left(0,0, A_{b}\right) \\
\mathbf{A}_{E} & =0
\end{aligned}
$$

We choose as independent parameters the mass $m_{A}$ of the CP-odd Higgs boson, the ratio of Higgs vevs $\tan \beta=v_{2} / v_{1}$, the gaugino mass parameter $M_{2}$, the higgs(ino) mass parameter $\mu$, and the quantities $m_{0}, A_{t}$ and $A_{b}$ above.

We include one-loop radiative corrections and two-loop leading-log contributions to the Higgs mass matrices using the effective potential approach described in 99.

The neutralinos $\tilde{\chi}_{i}^{0}$ are linear combinations of the neutral gauginos $\tilde{B}, \tilde{W}_{3}$ and of the neutral higgsinos $\tilde{H}_{1}^{0}, \tilde{H}_{2}^{0}$. In this basis, their mass matrix

$$
\mathcal{M}_{\tilde{\chi}_{1,2,3,4}^{0}}=\left(\begin{array}{cccc}
M_{1} & 0 & -\frac{g^{\prime} v_{1}}{\sqrt{2}} & +\frac{g^{\prime} v_{2}}{\sqrt{2}} \\
0 & M_{2} & +\frac{g v_{1}}{\sqrt{2}} & -\frac{g v_{2}}{\sqrt{2}} \\
-\frac{g^{\prime} v_{1}}{\sqrt{2}} & +\frac{g v_{1}}{\sqrt{2}} & 0 & -\mu \\
+\frac{g^{\prime} v_{2}}{\sqrt{2}} & -\frac{g v_{2}}{\sqrt{2}} & -\mu & 0
\end{array}\right)
$$

can be diagonalized analytically to give four neutral Majorana states,

$$
\tilde{\chi}_{i}^{0}=N_{i 1} \tilde{B}+N_{i 2} \tilde{W}^{3}+N_{i 3} \tilde{H}_{1}^{0}+N_{i 4} \tilde{H}_{2}^{0}
$$


the lightest of which, to be called $\chi$, is then the candidate for the particle making up (at least some of) the dark matter in the universe.

We consider only models that satisfy all accelerator constraints on supersymmetric particles and couplings; in particular, the measurement of the $\mathrm{b} \rightarrow \mathrm{s} \gamma$ process at the Cornell accelerator [10], which provides important bounds. However, to show the impact of present dark matter searches, we plot also models which are excluded by these nonaccelerator searches.

\section{Relic Density and Capture Rates}

For each model allowed by the accelerator constraints we calculate the relic density of neutralinos $\Omega_{\chi} h^{2}$. We use the formalism in Ref. [11] to carefully treat resonant annihilations and threshold effects, keeping finite widths of unstable particles, including all two-body annihilation channels of neutralinos. The annihilation cross sections were derived using a novel helicity projection technique [12], and were checked against published results for several of the subprocesses.

In this letter, we keep only models in which the neutralino density does not overclose the Universe and in which neutralinos can make up the totality of the galactic dark matter. Namely we require $\Omega_{\text {gal.DM }} h^{2}<\Omega_{\chi} h^{2}<1$, where (somewhat arbitrarily) we choose $\Omega_{\text {gal.DM }} h^{2}=0.025$. We have adopted a local dark matter density of $0.3 \mathrm{GeV} / \mathrm{cm}^{3}$.

The capture rate in the Earth is dominated by scalar interactions, and presents kinematic enhancements whenever the mass of the neutralino almost matches one of the heavy elements in the Earth. For the Sun, both axial interactions with hydrogen and scalar interactions with heavier elements are important. For both the Sun and the Earth we use the convenient approximations available in [13].

\section{Muon fluxes from neutralino annihilations}

Neutralinos in the core of the Sun and/or Earth can annihilate to a fermion-antifermion pair, to gauge bosons, Higgs bosons and gluons $\left(\chi \chi \rightarrow \ell^{+} \ell^{-}, q \bar{q}, g g, q \bar{q} g, W^{+} W^{-}\right.$, $\left.Z^{0} Z^{0}, Z^{0} H^{0}, W^{ \pm} H^{\mp}, H^{0} H^{0}\right)$. These annihilation products will hadronize and/or decay, eventually producing high energy muon neutrinos. Since the rate of muons in a neutrino telescope is approximately proportional to the neutrino energy squared (since both the cross section and the muon range are approximately proportional to the energy), the annihilation channels with the hardest neutrino spectra will be the most important, i.e. $W^{+} W^{-}, Z^{0} Z^{0}, t \bar{t}$, etc. In our calculation of the neutrino fluxes we have however included all annihilation channels (except gluons since they give very soft neutrino spectra).

With Monte Carlo simulations we have considered the whole chain of processes from

the annihilation products in the core of the Sun or the Earth to detectable muons at 
the surface of the Earth [14]. We have performed a full Monte Carlo simulation of the hadronization and decay of the annihilation products using JETSET 7.4 [15], of the neutrino interactions on their way out of the Sun and of the charged-current neutrino interactions near the detector using PYTHIA 5.7 [15], and finally of the multiple Coulomb scattering of the muon on its way to the detector using distributions from Ref. [16].

With respect to calculations using Ref. [17] (e.g. Ref. [18]), this Monte Carlo treatment of the neutrino propagation through the Sun bypasses simplifying assumptions previously made, namely neutral currents are no more assumed to be much weaker than charged currents and energy loss is no more considered continuous. For details on this treatment, see Ref. [14, 19].

The muon flux at a detector has been simulated for a set of neutralino masses $\left(m_{\tilde{\chi}}=\right.$ $10,25,50,80.3,91.2,100,150,176,200,250,350,500,750,1000,1500,2000,3000$ and $5000 \mathrm{GeV})$ and annihilation channels $\left(c \bar{c}, b \bar{b}, t \bar{t}, \tau^{+} \tau^{-}, W^{+} W^{-}\right.$and $\left.Z^{0} Z^{0}\right)$. For each mass and channel, $2.5 \times 10^{5}$ annihilations have been simulated. For masses other than those simulated, an interpolation is performed and the muon flux from channels other than those listed above are easily calculated since all other annihilation products decay to these particles (lighter quarks, electrons and muons do not contribute significantly to the neutrino flux). For the Higgs bosons, which decay in flight, an integration over the angle of the decay products with respect to the direction of the Higgs boson is performed. Given the branching ratios for different annihilation channels it is then straightforward to obtain the muon flux above any given energy threshold and within any angular region around the Sun or the center of the Earth.

\section{Indirect detection rates}

To illustrate the potential of neutrino telescopes for discovery of dark matter through neutrinos from the Earth or the Sun, we present the results of our full calculation. We show together results obtained with one 'normal' scan in the parameter space letting $\mu, M_{2}, \tan \beta, m_{A}, m_{0}, A_{b}$ and $A_{t}$ vary at random between generous bounds and one 'special' scan where we have been more restrictive on the $A$ mass,

$$
\left\{\begin{array} { l } 
{ \mu \in [ - 5 0 0 0 , 5 0 0 0 ] \mathrm { GeV } } \\
{ M _ { 2 } \in [ - 5 0 0 0 , 5 0 0 0 ] \mathrm { GeV } } \\
{ \operatorname { t a n } \beta \in [ 1 . 2 , 5 0 ] } \\
{ m _ { A } \in [ 0 , 1 0 0 0 ] \mathrm { GeV } } \\
{ m _ { 0 } \in [ 1 0 0 , 3 0 0 0 ] \mathrm { GeV } } \\
{ A _ { b } \in [ - 3 , 3 ] m _ { 0 } } \\
{ A _ { t } \in [ - 3 , 3 ] m _ { 0 } }
\end{array} \quad \text { 'normal' } \quad \left\{\begin{array}{l}
\mu \in[-5000,5000] \mathrm{GeV} \\
M_{2} \in[-5000,5000] \mathrm{GeV} \\
\tan \beta \in[1.2,50] \\
m_{A} \in[0,150] \mathrm{GeV} \\
m_{0} \in[100,3000] \mathrm{GeV} \\
A_{b} \in[-3,3] m_{0} \\
A_{t} \in[-3,3] m_{0}
\end{array}\right.\right.
$$

We recall that the density of points in the figures reflects our choices for scanning the parameter space, and is therefore subjective (for a discussion on this see Ref. [8]). 
In Fig. 1 we show our predictions for the indirect detection rates as a function of neutralino mass. The horizontal lines are the best present limits for indirect searches and come from the Baksan detector [6]. The limits are $\Phi_{\mu}^{\text {Earth }}<2.1 \times 10^{-14} \mathrm{~cm}^{-2}$ $\mathrm{s}^{-1}$ and $\Phi_{\mu}^{\text {Sun }}<3.5 \times 10^{-14} \mathrm{~cm}^{-2} \mathrm{~s}^{-1}$ at $90 \%$ confidence level and integrated over a half-angle aperture of $30^{\circ}$ with a muon energy threshold of $1 \mathrm{GeV}$. Quite a few models with high neutrino rates are already ruled out by the Baksan data. These models have large $\tan \beta \gtrsim 30$, low $H_{2}$ mass, and large mixing for stop squarks. In Fig. 1 it can also be seen that a neutrino telescope of an area around $1 \mathrm{~km}^{2}$, which is a size currently being discussed, would have a large discovery potential for supersymmetric dark matter. We remind that, after subtraction of the atmospheric neutrino fluxes (by means of an on-source off-source difference, e.g.), the remaining background is due to high-energy neutrinos produced by cosmic ray collisions in the solar atmosphere, and is at the level of 15 muons $(>1 \mathrm{GeV}) / \mathrm{km}^{2} / \mathrm{yr}$ [20].

In Fig. 2 we show the muon rates versus $\Omega_{\chi} h^{2}$. The general trend is that large $\Omega_{\chi} h^{2}$ corresponds to lower rates, as would be expected from crossing symmetry between annihilation and scattering cross sections. Note, however, the large spread of the predicted rates for a given value of $\Omega_{\chi} h^{2}$.

In Fig. 3 a comparison is made between the predicted rates from the Earth and from the Sun for the same set of models. In the region yet to explore, the rate from the Sun is generally larger than the rate from the Earth.

In Fig. 团 the indirect detection rate is compared to the direct detection rate in ${ }^{76} \mathrm{Ge}$. As can be seen, there is a correlation between the two, although for the Sun it is not as strong as for the Earth, where a high capture rate is due to a large scalar cross section, which also means a high rate in Germaniums. Without forgetting the huge spread, we see that for a given factor of improvement in sensitivity, indirect detection from the Sun generally scores better than direct detection, which in turn generally scores better than indirect detection from the Earth.

Note that the muon rates in real experiments may be significantly lower (by as much as an order of magnitude for neutralinos in the lower mass range) due to the need to impose a higher energy threshold for the signal than assumed here. We have taken 1 $\mathrm{GeV}$ for neutrino telescopes which is true for a small scale detector like Baksan; for a kilometer-scale array it is more likely to be tens of GeV. Likewise, the Germanium rate given is the integrated rate from zero recoil energy to the kinematical limit. Present-day detectors typically only sample a small range of recoil energies.

Since the special scan employs fairly low values of the $A$ mass, one may wonder whether the $A$ or the lightest Higgs boson $H_{2}$ would be light enough to be discovered or excluded at LEP2. In particular, one could expect this for the models that give

\footnotetext{
${ }^{\S}$ Recently, a paper appeared (V.A. Bednyakov et al., hep-ph/9606261), where no high Germanium rates were found in a variant of the special scan employed here. This seems to be due to their use of a more restricted model, imposing unification conditions of the scalar mass parameters at the GUT scale, which we do not.
} 
high neutrino rates, since a low Higgs mass gives a large spin-independent scattering cross section of (mixed) neutralinos and therefore large capture and annihilation rates. Using the expected exclusion limits for LEP2 given in Ref. 21] we find, indeed, that the models in our sample with the highest rates are within reach of LEP2, because of the large cross section for $e^{+} e^{-} \rightarrow A H_{2}$. Technically this is due to the fact that these models have a large value of the factor $\cos ^{2}(\beta-\alpha)$ that governs the $Z A H_{2}$ coupling (with $\alpha$ being the CP-even Higgs mixing angle). In Fig. 5 we show the indirect detection rates versus $m_{H_{2}}$ : the models that can be probed by LEP2 are shown in the upper part of the figure, the others in the lower part. We have used the combination of all four LEP experiments and assumed a total integrated luminosity of $150 \mathrm{pb}^{-1}$ at $192 \mathrm{GeV}$. We have also included the possible LEP2 lower limit of $95 \mathrm{GeV}$ on the mass of the chargino and the bounds coming from the $e^{+} e^{-} \rightarrow Z H_{2} \rightarrow Z b \bar{b}$ process. LEP2 will probe all models in our scan with $m_{H_{2}} \lesssim 90 \mathrm{GeV}$, and in particular most of the models already ruled out by Baksan. On the other hand, there are many models giving quite large indirect detection rates that cannot be probed by LEP2, but that would be accessible at a large neutrino telescope.

In conclusion, indirect dark matter searches and LEP2 probe complementary regions of the supersymmetric parameter space. Moreover, direct detection (see \&]) is reaching a sensitivity that allows some models to be excluded, with somewhat different characteristics than those probed by the other methods. This illustrates a nice com-

plementarity between direct detection, indirect detection and accelerator methods to bound or confirm the minimal supersymmetric standard model.

\section{Acknowledgments}

This work has been partially supported by the EC Theoretical Astroparticle Network under contract No. CHRX-CT93-0120 (Direction Générale 12 COMA). L.B. wants to thank the Swedish Natural Science Research Council (NFR) for support. P.G. is grateful to the organizers of the Uppsala Astroparticle Workshop, during which this work was completed.

\section{References}

[1] J. Silk, K. Olive and M. Srednicki, Phys. Rev. Lett. 55 (1985) 257.

T. Gaisser, G. Steigman and S. Tilav, Phys. Rev. D34 (1986) 2206.

B.A. Campbell, J. Ellis, K. Enqvist, D.V. Nanopoulos, J. Hagelin and K.A. Olive, Phys. Lett. B173 (1986) 270.

M. Srednicki, K. Olive and J. Silk, Nucl. Phys. B279 (1987) 804. 
K. Griest and S. Seckel, Nucl. Phys. B283 (1987) 681.

J. Hagelin, K. Ng and K. Olive, Phys. Lett. B180 (1987) 375.

K. Ng, K. A. Olive and M. Srednicki, Phys. Lett. B188 (1987) 138.

[2] K. Freese, Phys. Lett. B167 (1986) 295.

L. Krauss, M. Srednicki and F. Wilczek, Phys. Rev. D33 (1986) 2079.

T. Gaisser, G. Steigman and S. Tilav, Phys. Rev. D34 (1986) 2206.

[3] W.H. Press and D.N. Spergel, Astrophys. J. 296 (1985) 679.

[4] H. Goldberg, Phys. Rev. Lett. 50 (1983) 1419.

[5] IMB Collaboration: J.M. Losecco et al., Phys. Lett. B188 (1987) 388.

Kamiokande Collaboration: M. Mori et al., Phys. Lett. B205 (1988) 416.

G. Gelmini, P. Gondolo and E. Roulet, Nucl. Phys. B351 (1991) 623.

M. Kamionkowski, Phys. Rev. D44 (1991) 3021.

A. Bottino, V. de Alfaro, N. Fornengo, G. Mignola and M. Pignone, Phys. Lett. B265 (1991) 57.

Kamiokande Collaboration: N. Sato et al., Phys. Rev. D44 (1991) 2220; M. Mori et al., Phys. Lett. B270 (1991) 89; Phys. Lett. B289 (1992) 463; Phys. Rev. D48 (1993) 5505.

[6] M.M. Boliev et al (Baksan telescope), Nucl. Phys. B (Proc. Suppl.) 48 (1996) 83, TAUP 95, Toledo, Spain, 17-21 September 1996, Editor: A. Morales

[7] S.W. Barwick, in Trends in Astroparticle Physics, Stockholm, Sweden, 1994, eds. L. Bergström, P. Carlson, P.O. Hulth and H. Snellman, Nucl. Phys. (Proc. Suppl.) B43, (1995).

[8] L. Bergström and P. Gondolo, Astrop. Phys., in press, hep-ph/9510252.

[9] M. Carena, J.R. Espinosa, M. Quirós and C.E.M. Wagner, Phys. Lett. B355 (1995) 209.

[10] M.S. Alam et al. (CLEO Collaboration), Phys. Rev. Lett. 71 (1993) 674; Phys. Rev. Lett. 74 (1995) 2885.

[11] P. Gondolo and G. Gelmini, Nucl. Phys. B360 (1991) 145.

[12] P. Gondolo, in preparation.

[13] G. Jungman, M. Kamionkowski and K. Griest, Phys. Rep. 267 (1996) 195. 
[14] J. Edsjö, Diploma Thesis, Uppsala University preprint TSL/ISV-93-0091 (ISSN 0284-2769).

J. Edsjö, in Trends in Astroparticle Physics, Stockholm, Sweden, 1994, eds. L. Bergström, P. Carlson, P.O. Hulth and H. Snellman, Nucl. Phys. (Proc. Suppl.) B43 (1995) 265.

[15] T. Sjöstrand, Comp. Phys. Comm. 82 (1994) 74.

T. Sjöstrand, PYTHIA 5.7 and JETSET \%.4. Physics and Manual, CERNTH.7112/93, hep-ph/9508391 (revised version).

[16] Particle Data Group, Phys. Rev. D50 (1994) 1173.

[17] S. Ritz and D. Seckel, Nucl. Phys. B304 (1988) 877.

[18] G.F. Giudice and E. Roulet, Nucl. Phys. B316 (1989) 429.

F. Halzen, T. Stelzer and M. Kamionkowski, Phys. Rev. D45 (1992) 4439.

M. Drees, G. Jungman, M. Kamionkowski and M.M. Nojiri, Phys. Rev. D49 (1994) 636.

R. Gandhi, J.L. Lopez, D.V. Nanopoulos, K. Yuan and A. Zichichi, Phys. Rev. D49 (1994) 3691.

A. Bottino, N. Fornengo, G. Mignola and L. Moscoso, Astropart. Phys. 3 (1995) 65 .

G. Jungman and M. Kamionkowski, Phys. Rev. D51 (1995) 328.

V. Berezinsky, A. Bottino, J. Ellis, N. Fornengo, G. Mignola and S. Scopel, hepph/9603342.

[19] J. Edsjö and P. Gondolo, Phys. Lett. B357 (1995) 595.

[20] D. Seckel, T. Stanev, and T. Gaisser, Ap. J. 382 (1991) 652.

[21] E. Accomando et al, "Higgs Physics at LEP2", hep-ph/9602250, to appear in Vol. 1, "Report of the Workshop on Physics at LEP2", G. Altarelli, T. Sjöstrand and F. Zwirner (eds), CERN 96-01. 


\section{Figures}

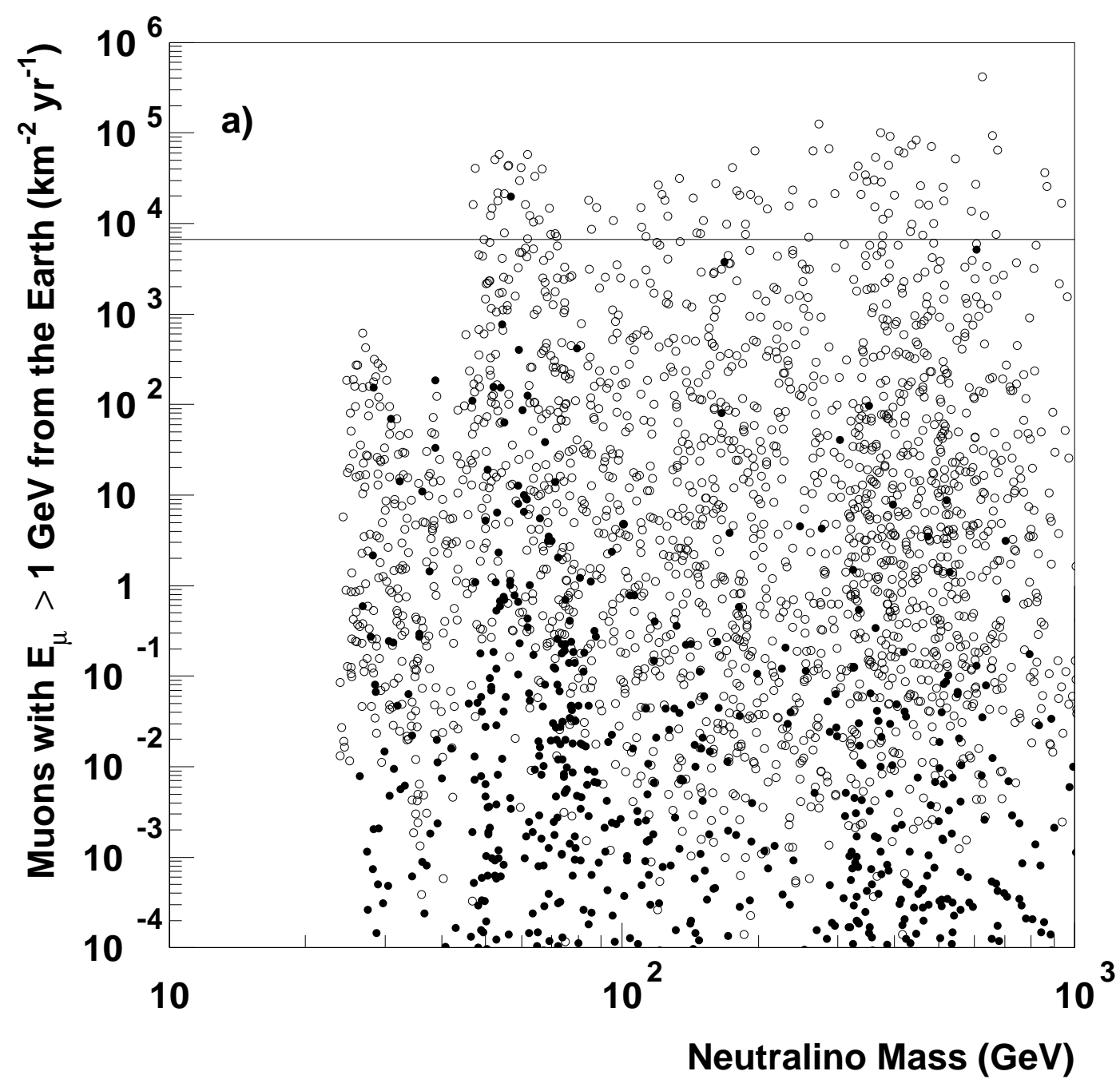

Figure 1: The indirect detection rates from neutralino annihilations in a) the Earth and b) the Sun versus the neutralino mass. The horizontal line is the Baksan limit [6]. Filled points are from a 'normal' scan and open points are from a 'special' scan with $m_{A}<150$ $\mathrm{GeV}$. 


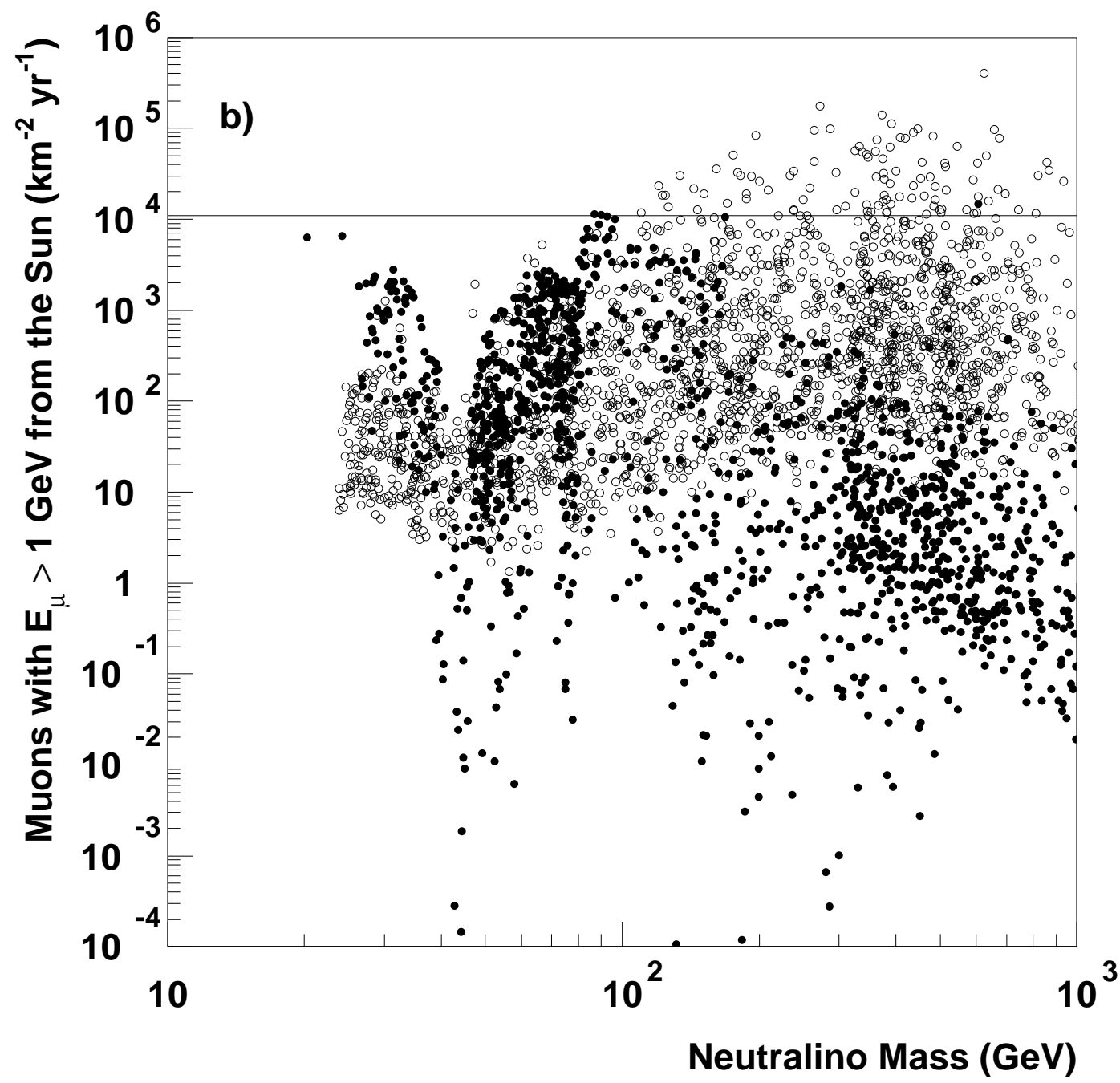




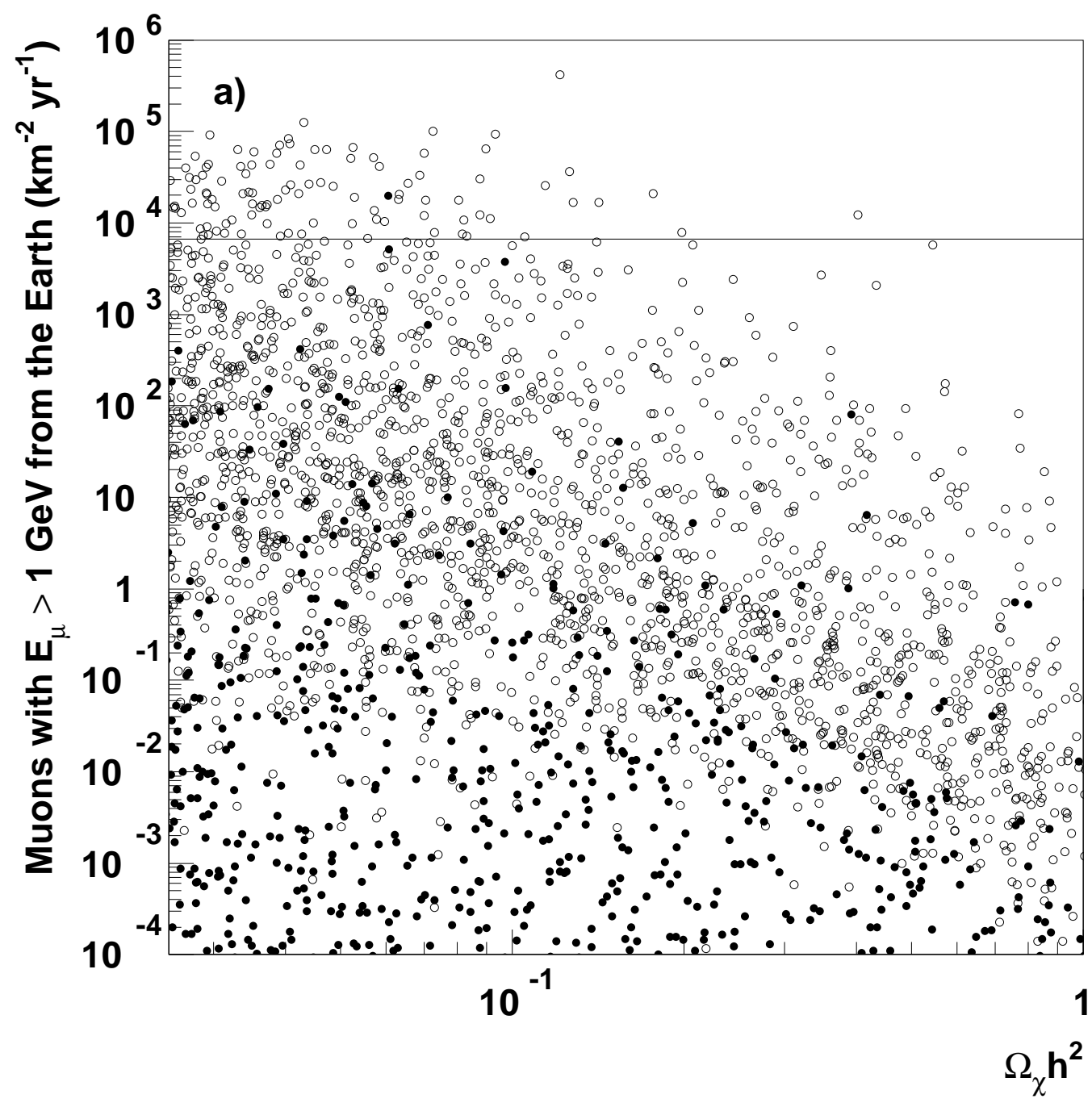

Figure 2: The indirect detection rates from neutralino annihilations in a) the Earth and b) the Sun versus $\Omega_{\chi} h^{2}$. The horizontal line is the Baksan limit [6]. Filled points are from a 'normal' scan and open points are from a 'special' scan with $m_{A}<150 \mathrm{GeV}$. 


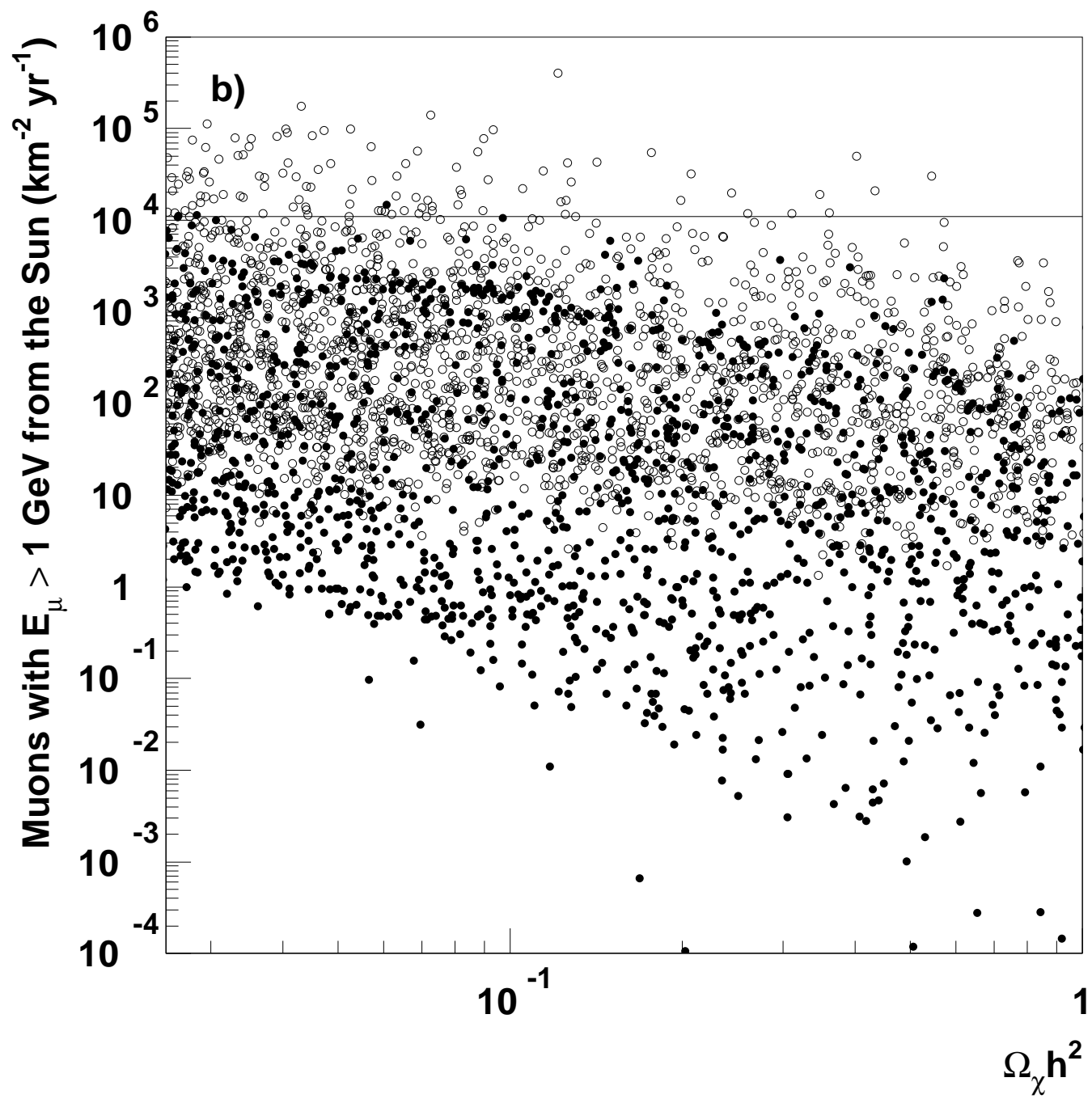




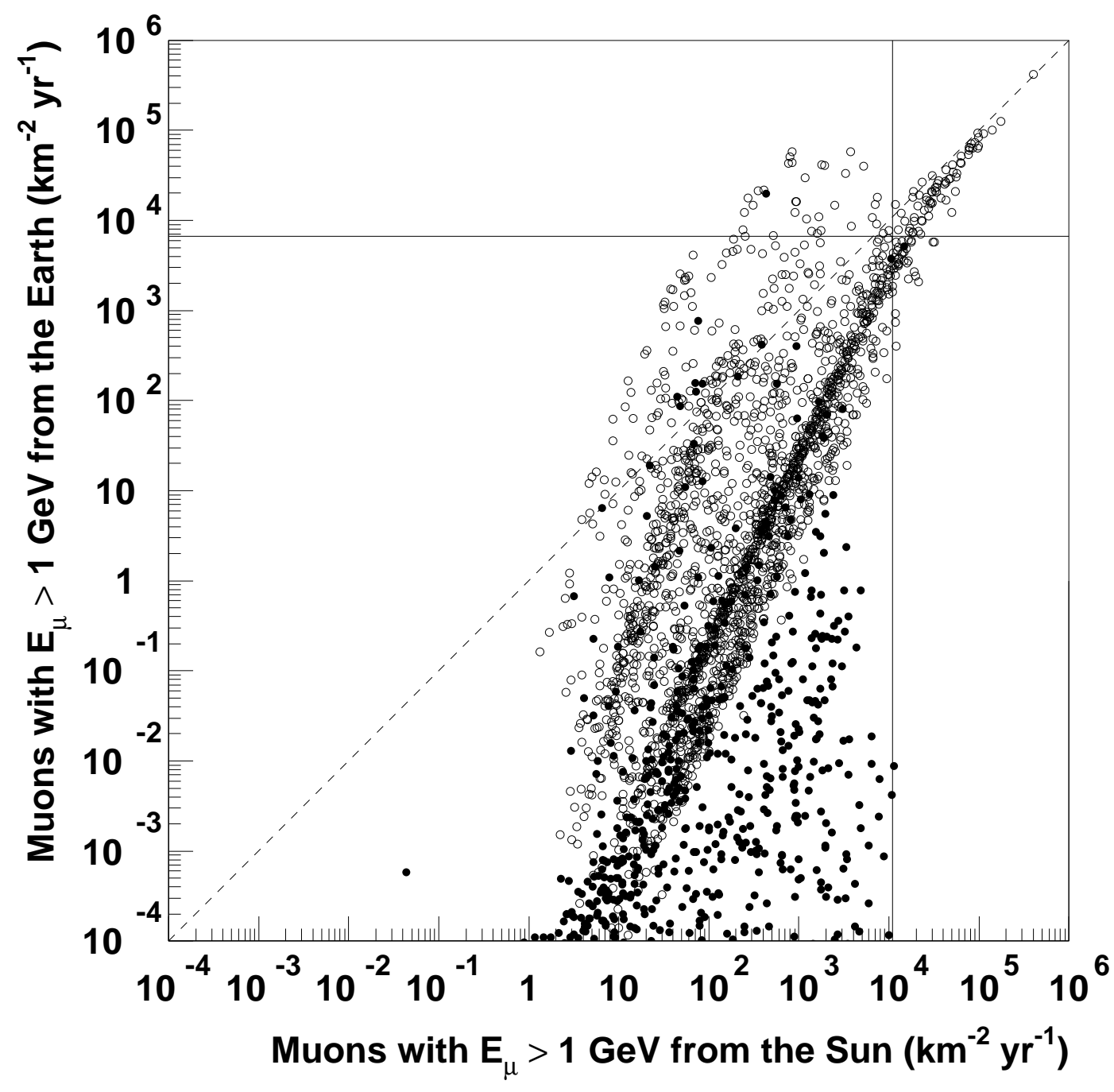

Figure 3: The indirect detection rates in the Earth versus the indirect detection rates in the Sun. The horizontal and vertical lines are the Baksan limits [6]. The dashed line, indicating equal rates, is shown just for convenience. Filled points are from a 'normal' scan and open points are from a 'special' scan with $m_{A}<150 \mathrm{GeV}$. 


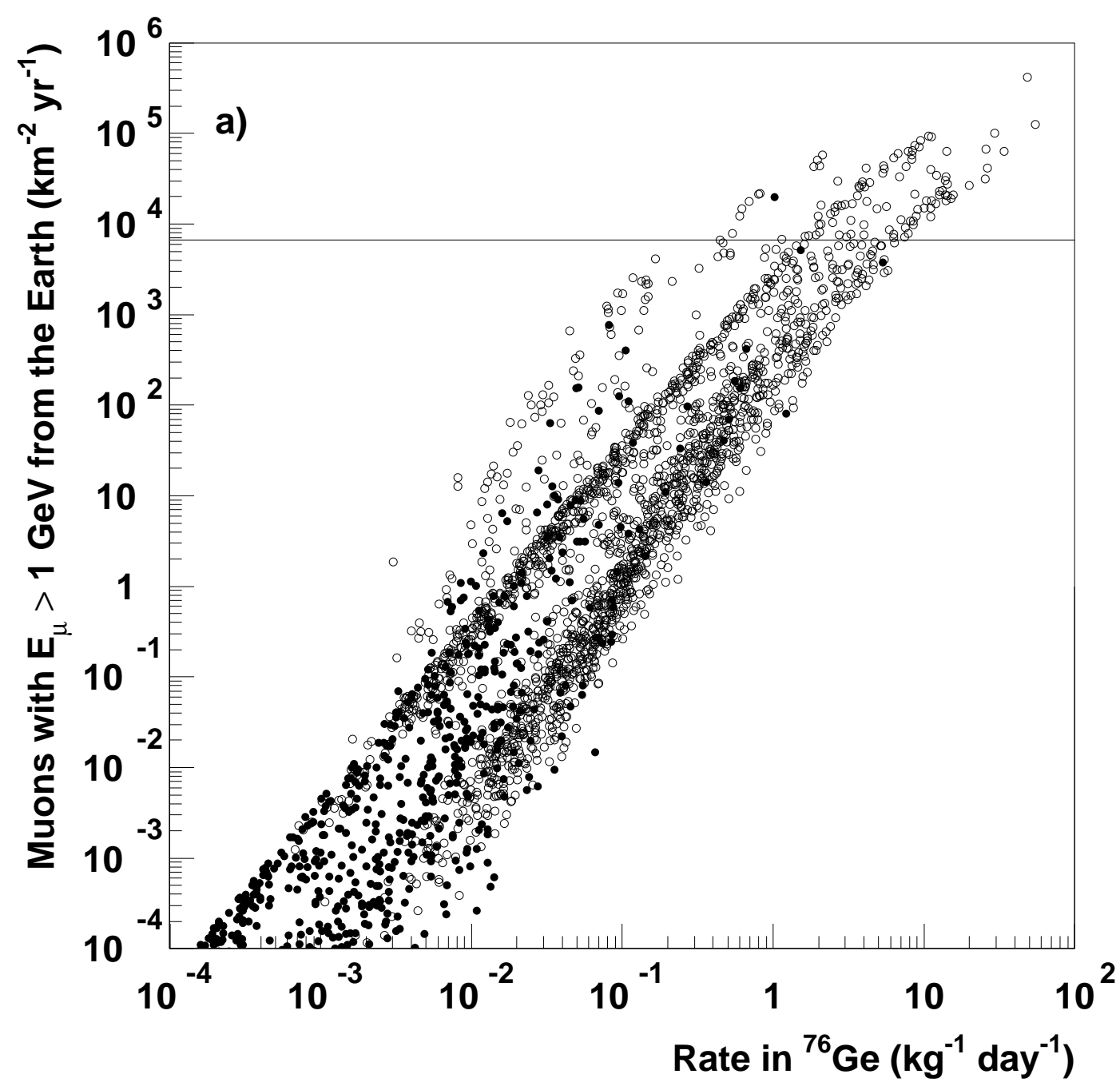

Figure 4: The indirect detection rates from neutralino annihilations in a) the Earth and b) the Sun versus the direct detection rate in ${ }^{76} \mathrm{Ge}$. The horizontal line is the Baksan limit [6]. Filled points are from a 'normal' scan and open points are from a 'special' scan with $m_{A}<150 \mathrm{GeV}$. 


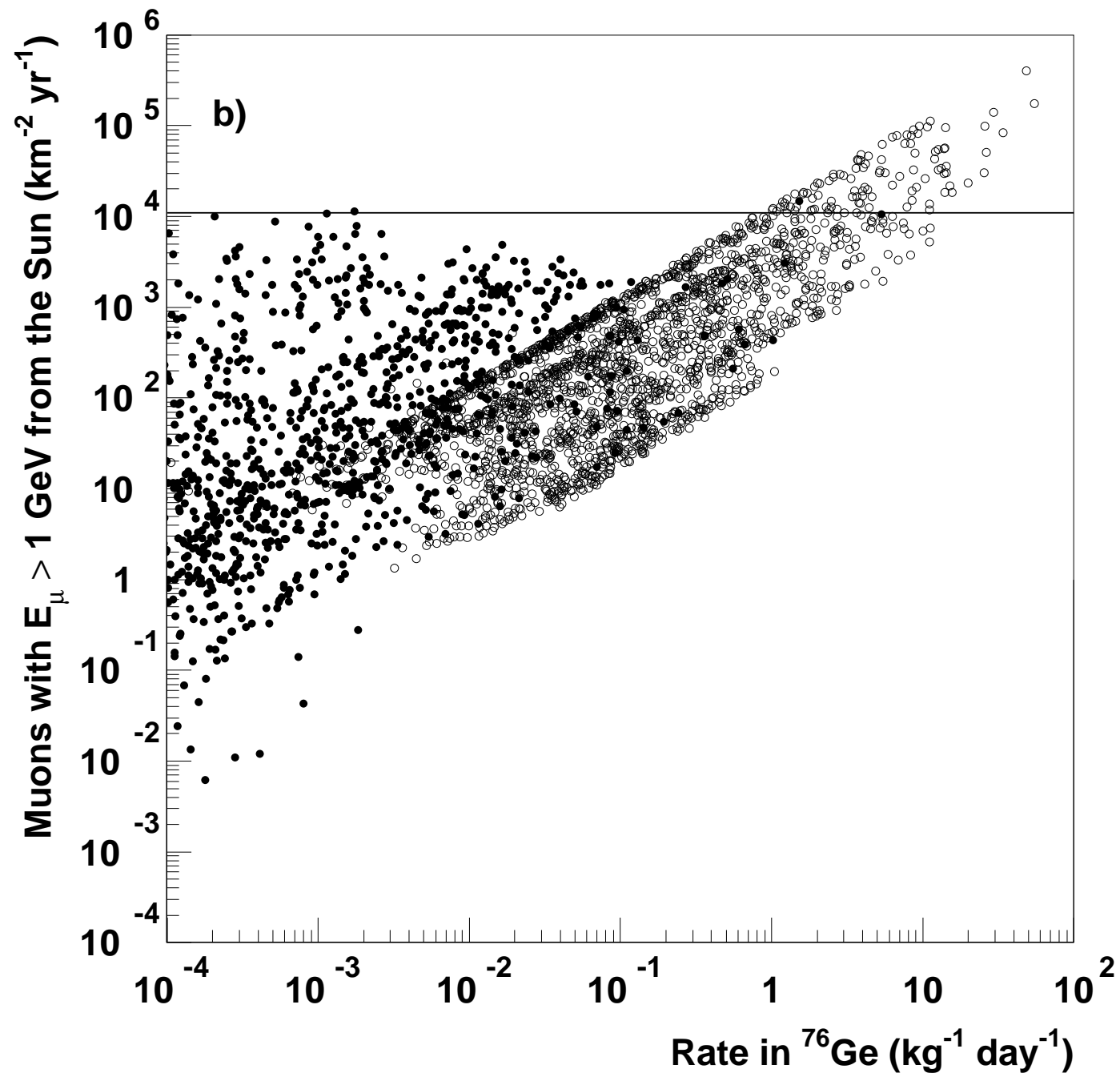




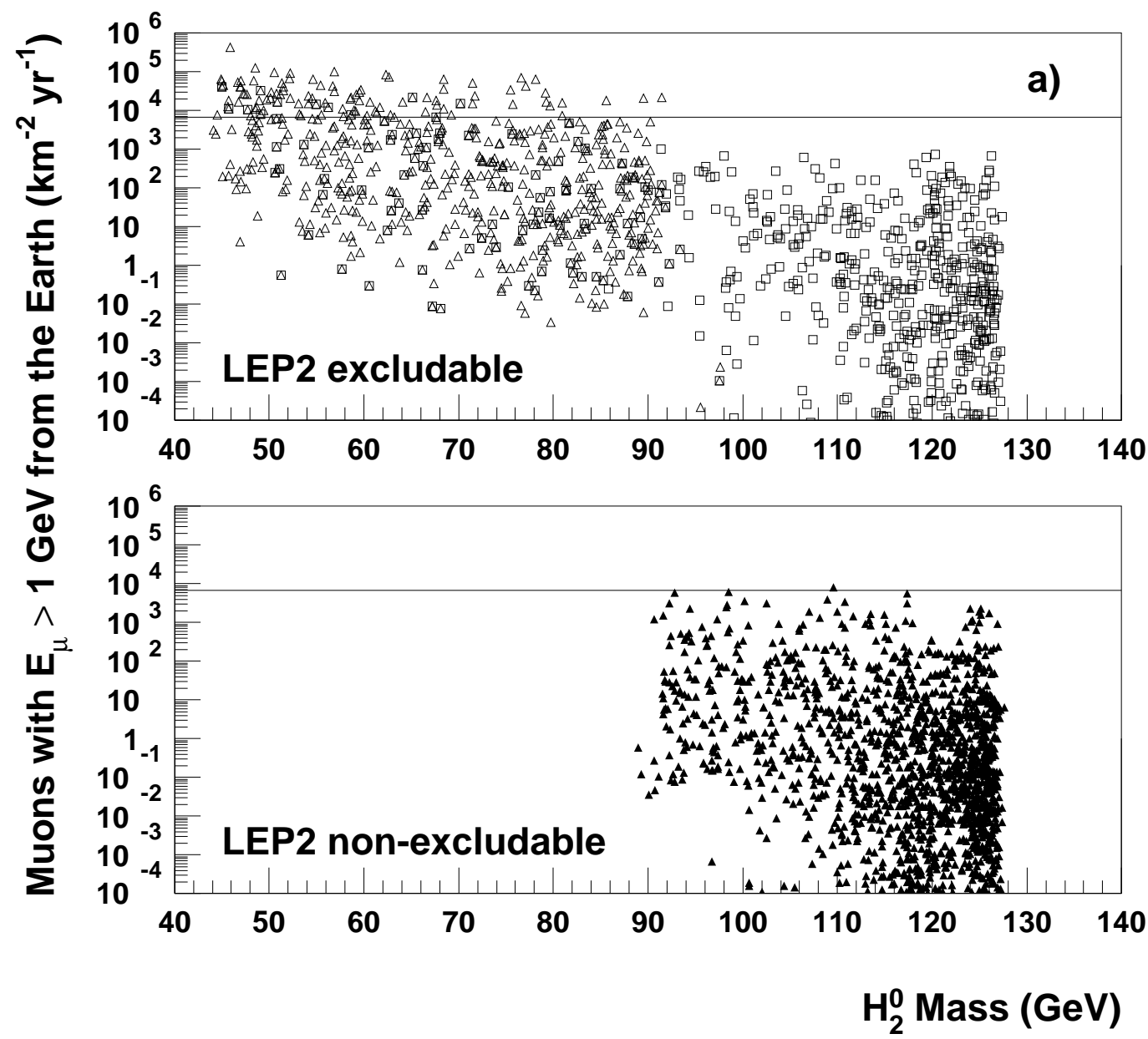

Figure 5: The indirect detection rates from neutralino annihilations in a) the Earth and b) the Sun versus $m_{H_{2}}$. The horizontal line is the Baksan limit [6]. The upper part are models which can be excluded at LEP2 after $150 \mathrm{pb}^{-1}$ of running at $192 \mathrm{GeV}$ and the lower part are models which cannot be excluded by LEP2. The open triangles are models that can be excluded due to no Higgs discovery and the open squares are models that can be excluded due to no chargino discovery. 


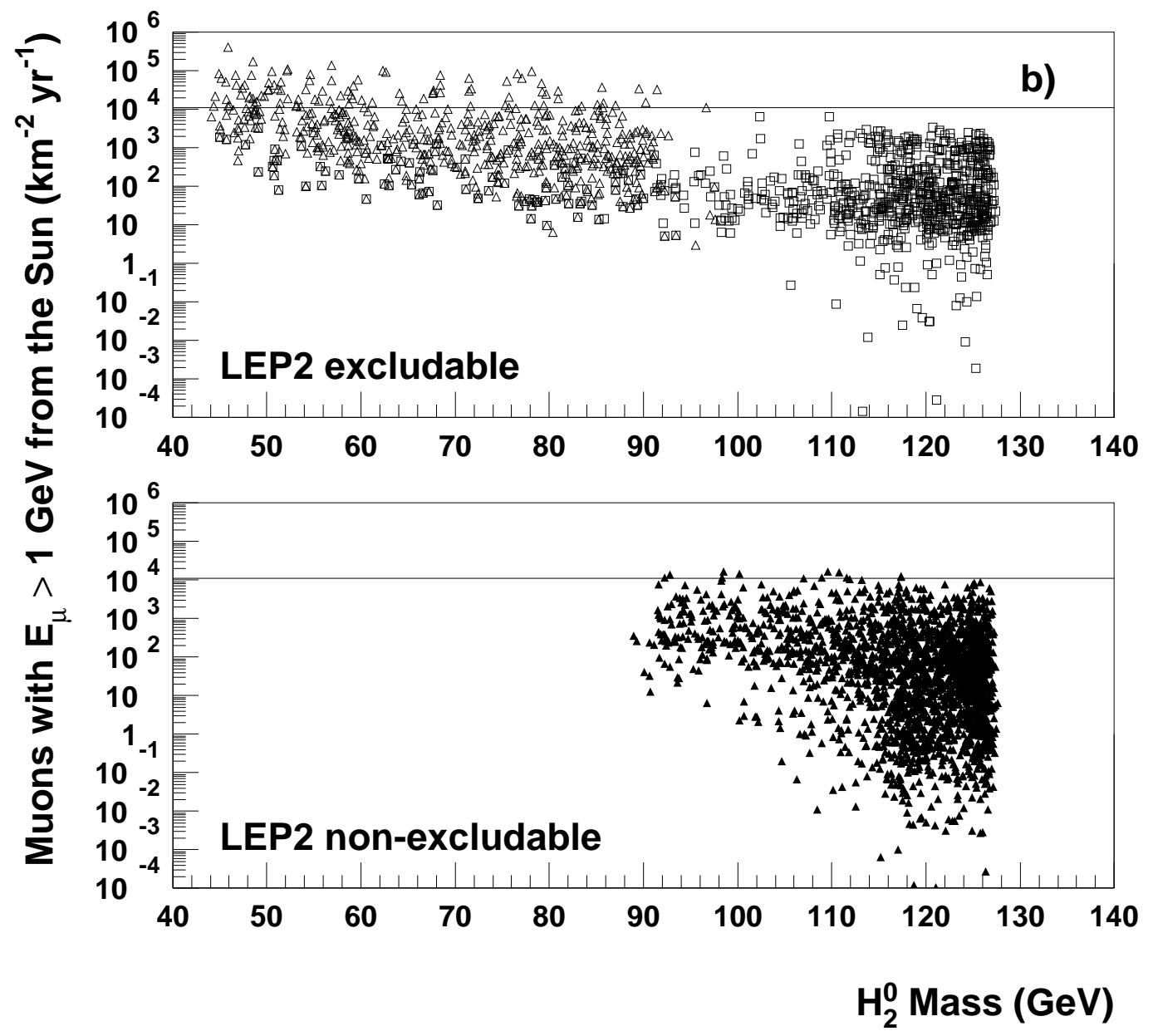

